\title{
E-Multidisciplinary Oncology Conference for Liver Cancer as a Technology and Method to Optimize Personalization and Outcomes in the Peripheries
}

\author{
Constantinos S. MAMMAS ${ }^{\mathrm{a}, 1}$ and Adamantia S. MAMMA ${ }^{\mathrm{a}}$ \\ aProgram of Excellence 2014-16-Siemens Program for Greece
}

\begin{abstract}
Aim: To search feasibility and reliability of Telemedicine Systems (TS) in the remote multidisciplinary oncology conference for decision making and treatment of liver lesions. Material and Methods: By an experimental TS, twenty six $(n=26)$ specialists based on a series of five $(N=5)$ simulated remote image examinations, assessed sensitivity-specificity of the remote examination of the Liver (L) for neoplastic diseases and damages (Virtual Examination=VE). Results: Analysis showed: injuries (sensitivity $=96 \%$ ), injuries of the capsula (sensitivity $=91.7 \%$ ), hematomas (sensitivity $=91,7 \%$ ), non-neoplastic diseases (specificity $=100 \%$ ), neoplastic diseases (sensitivity $=100 \%$ ). Conclusion: The VE of the (L) in combination with high-tech visualization and multimedia and the remote participation of liver surgical oncology, oncology, radiology, pathology and cytology experts composes a feasible and reliable e-Multidisciplinary Oncologic Conference for a Personalized and Optimum Decision Making and Treatment in Liver Cancer.
\end{abstract}

Keywords. Tele-Medicine, Tele-pathology, Tele-radiology, Liver Cancer, EMultidisciplinary Oncologic Conference

\section{Introduction}

The optimal management of patients with liver cancer (LC) requires the expertise of specialists from different disciplines. This has led to the evolution of multidisciplinary teams (MDTs), allowing all key professionals to jointly discuss individual patients and to contribute independently to clinical decisions. However, LC MDTs in different regions in the periphery and countries are scarce [1,2]. The project searches feasibilityreliability of Telemedicine Systems (TS) integrated with Multimedia Systems (MS) for remote e-Multidisciplinary Oncology Conference in LC interrelated with Teleradiology (TRE) and Telepathology (TPE) evaluation of the patients and the liver (L) for optimum decision making and surgical planning $[3,4,5]$.

${ }^{1}$ Corresponding Author, Constantinos St. Mammas MD, MSc, PhD, 9, Kononos Ave., Pagrati, Athens, Greece; Email: csmammas@med.uoa.gr. 


\section{Material and Methods}

Experimentation included.

The development of an OTE-TS similar Experimental TS (Exp.-TS) for the simulation of the integrated TS, MS and AI based TRE and TPE virtual examination of the patient and the liver viscera remotely (pn 1009078, 34931, 34932, 34933) (Table 1).

Table 1. Comparison of the Modules between OTE-TS and Exp.-TS

\begin{tabular}{ccc}
\hline Modules & OTE-TS & EXP.-TS \\
\hline a. Medical record process & + & + \\
b. Examinations results. & + & + \\
c. Capture scanning and imaging. & + & + \\
d. DICOM and PACS vision. & + & + \\
e. Real-time tele-conference & + & + \\
f. Chat and whiteboard facilities. & + & + \\
g. Application sharing. & + & + \\
h. Tele-secretary facilities. & + & + \\
j. Tele-Mentoring facilities & + & + \\
i. Telecommunication net & ISDN based & + \\
k. Multimedia System & + & + \\
1.AI computation system & - & + \\
\hline
\end{tabular}

Simulation of the TRE of (Ls) on 15 abdominal MR digital images taken by the ACS-NT GYROSCAN MRI POWERTRACK 6000, 1.5T, (by PHILIPS) and projected on the Exp.-TS in the intranet of the Department of Radiology of the Aretaieion University Hospital for examination of damages and lesions by a radiologist (2012) (Figure 1).

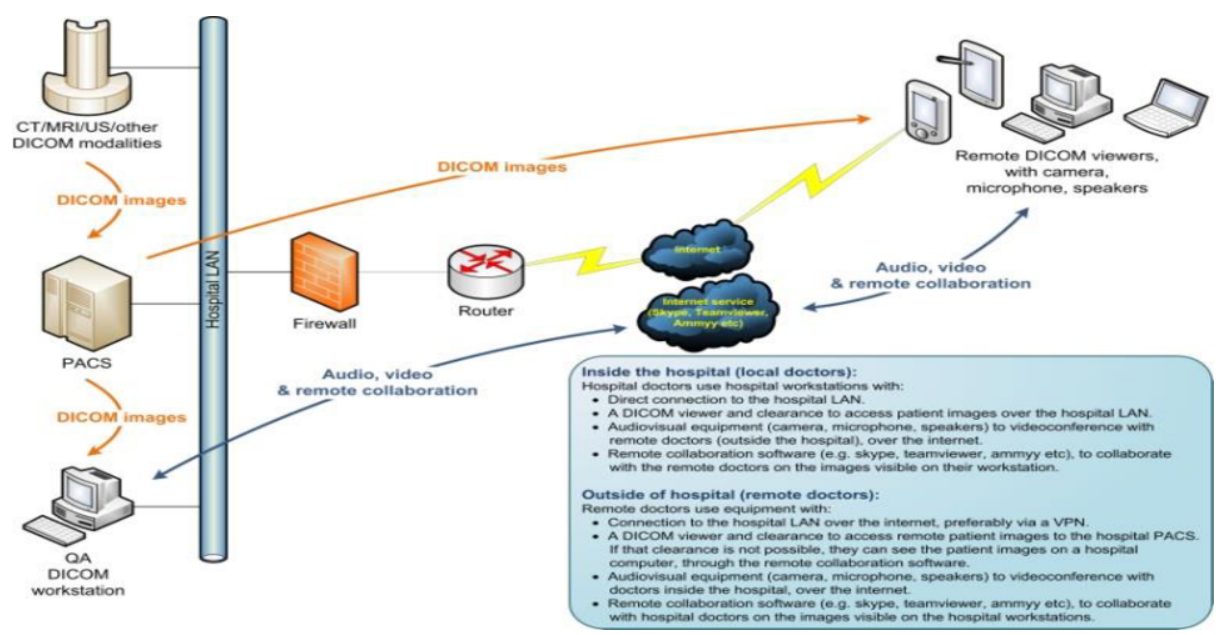

Figure 1. Collaboration between the hospital in the periphery and the Multidisciplinary Team for the remote examination of the patient's medical record and its medical data focused on Liver Cancer for the realization of the e-Multidisciplinary Oncology Conference for remote decision making and surgical planning via internet (DICOM and PACS based remote digital visualization of the necessary radiological examinations). 
Experimental simulation of the remote virtual evaluation of the (L) upon $n=5$ digital histological images by 26 specialists in the intranet of the department of Pathology of the Medical School of Athens (2013).

Sensitivity-specificity analysis of the results by using the SPSS statistical software (version 17.0).

\section{Results}

All examiners defined (L) and diagnosed damages and lesions (Tables 2, 3, 4).

Table 2. Simulated TRE of the patients' (L)

\begin{tabular}{lcccccccc}
\hline & N & TP & FP & FN & TN & SENSITIVITY & SPECIFICITY & ACCURACY \\
\hline Damages and Lesions & 15 & 4 & 0 & 0 & 11 & $\mathbf{1 0 0 , 0}$ & $\mathbf{1 0 0 , 0}$ & $\mathbf{1 0 0 , 0}$ \\
\hline $\begin{array}{l}\text { N=Number of images given for examination, } \\
\text { Negative, Sensitivity(\%), Specificity }(\%) \text {, Diagnostic accuracy }(\text { Efficiency)( } \% \text {.) }\end{array}$ &
\end{tabular}

Table 3. Simulated TPE of the patients' (L)

\begin{tabular}{cccccccccc}
\hline & N & A & TP & FP & FN & TN & SENSITIVITY & SPECIFICITY & ACCURACY \\
\hline Damages and Lesions & 26 & 26 & 24 & 0 & 1 & 0 & $\mathbf{9 6 , 0}$ & - & $\mathbf{9 6 , 0}$ \\
\hline
\end{tabular}

$\mathrm{N}=$ Number of Photos given for examination, $\mathrm{A}=$ Number of Answers after Examination, $\mathrm{TP}=\mathrm{True}$ Positive, $\mathrm{FP}=\mathrm{False}$ Positive, FN=False Negative, TN=True Negative, Sensitivity (\%), Specificity(\%), Diagnostic accuracy (Efficiency)( \%.)

Table 4. Integrated TRE and TPE of the patients' (L)

\begin{tabular}{lcccccccc}
\hline & N & TP & FP & FN & TN & SENSITIVITY & SPECIFICITY & ACCURACY \\
\hline Damages and Lesions & 41 & 28 & 0 & 1 & 11 & $\mathbf{9 6 , 6}$ & $\mathbf{1 0 0 , 0}$ & $\mathbf{9 7 , 5}$ \\
\hline $\begin{array}{l}\text { N=Number of images given for examination, } \\
\text { Negative, Sensitivity(\%), Specificity(\%), Diagnostic accuracy (Efficiency)(\%.). }\end{array}$
\end{tabular}

\section{Discussion}

The abovementioned very promising results confirmed clinically in the periphery of Greece with a clinical case of a female patient which had suffered from a right renal cancer that had been treated in the past but now suffered from an exacerbation characterized with a right lobe liver mass (July 2021). The patient treated with a surgical intervention based on the decision of the remote e-Multidisciplinary Oncology Conference in Liver Cancer using the above described technology and method focusing on tele-radiology. With regard to safety issues RH Morgan [6] proposed the Virtual private networks (VPN) as adequate solutions for authentication, access control and confidentiality. The secure hypertext transfer protocol (https) can be used to encrypt for web distribution. A public key infrastructure (PKI) solves all the issues mentioned above. Clinical efficacy research protocols for further study of the remote e-Multidisciplinary Oncology Conference in Liver Cancer is of high priority [7,8]. 


\section{Conclusion}

The VE of the (L) in combination with high-tech visualization and multimedia technology and the remote participation of liver surgical oncology experts for the realization of the e-Multidisciplinary Oncologic Conference seems feasible and reliable to optimize liver cancer personalized decision making and then multidisciplinary management in high volume liver units for the patients in the periphery of Hellas.

\section{References}

[1] Siddique O, Yoo ER, Perumpail RB et al.:The importance of a multidisciplinary approach to hepatocellular carcinoma. Journal of Multidisciplinary Healthcare 2017 Mar; 10:95-100.

[2] Couri T, Pillai A. Goals and targets for personalized therapy for HCC. Hepatol Int. 2019 Mar;13(2):125137.

[3] Mammas CS, Mamma AS, Papaxoinis G, Georgiou I. AI supported decision making and planning in 40 breast surgery operations. In:Aristoteleio University of Thessaloniki Editor. Proceedings of the 32nd Panhellenic Congress of Surgery and International Forum; 2021 Jun 9-12; Thessaloniki:p.237.

[4] Mammas CS, Mandellos G, Economou GP, Lymberopoulos D. Structuring Expert-Leaded Medical Protocols for Telemedicine Systems. In: IEEE Editor. Proceedings of the 23rd Annual International Conference of the IEEE Engineering in Medicine and Biology Society; 2001 Oct 25-28; Constantinople: p.p.3529-32.

[5] Karavatselou E, Economou GP, Chassomeris C, Daneli V, Lymperopoulos D. OTE-TS-a new valueadded telematics service for telemedicine applications. IEEE Transactions on Information Technologies in Biomedicine. 2001 Sep;5(3):210-24.

[6] Morgan RH. Computer network security for the radiology enterprise Computer network. Radiology. 2001 Aug;220(2):303-09.

[7] Furness PN, Bamford WM. Tele-pathology. Curr Diag Pathol. 2001 Dec;7:281-291.

[8] Coiera E, editor. The Internet and Telemedicine. In: Coiera E, editor. Guide to Medical Informatics. 2nd ed. New York: CHM; c2007. p.408. 